\title{
BMJ Open Efficacy of umbilical cord mesenchymal stem cell transfusion for the treatment of severe AKI: a protocol for a randomised controlled trial
}

\author{
Yuanjun Yang (D) , Jianjun Gao (1) , Siyang Wang, Wenjuan Wang, Fang-Lei Zhu, \\ Xiaolong Wang, Shuang Liang, Zhe Feng, Shupeng Lin, Li Zhang, Xiangmei Chen, \\ Guangyan Cai (i)
}

To cite: Yang Y, Gao J, Wang $\mathrm{S}$, et al. Efficacy of umbilical cord mesenchymal stem cell transfusion for the treatment of severe AKI: a protocol for a randomised controlled trial. BMJ Open 2022;12:e047622. doi:10.1136/ bmjopen-2020-047622

- Prepublication history and additional supplemental material for this paper are available online. To view these files, please visit the journal online (http://dx.doi.org/10.1136/ bmjopen-2020-047622).

Received 05 December 2020 Accepted 26 January 2022

\section{Check for updates}

(C) Author(s) (or their employer(s)) 2022. Re-use permitted under CC BY-NC. No commercial re-use. See rights and permissions. Published by BMJ.

Department of Nephrology, Chinese PLA General Hospital, Beijing, China

Correspondence to Professor Guangyan Cai; caiguangyan@sina.com

\section{ABSTRACT}

Introduction Acute kidney injury (AKI) is a common and severe clinical problem that is associated with high mortality, a long hospital stays and high healthcare resource consumption. Approximately a quarter of AKI survivors will develop chronic kidney disease. Mesenchymal stem cells (MSCs) are multipotent stem cells with antiapoptotic, immunomodulatory, antioxidative and proangiogenic properties. Therefore, MSCs have been considered as a potential new therapy for the treatment of AKI. Several clinical trials have been performed, but the results have been inconsistent. This trial investigated whether MSCs can improve renal recovery and mortality in patients with severe AKI.

Methods and analysis One hundred subjects suffering from severe AKI will participate in this patient-blinded, randomised, placebo-controlled, parallel design clinical trial. Participants will be randomly assigned to receive two doses of MSCs or placebo (saline) on days 0 and 7. Urinary biomarkers of renal injury and repair will be measured using commercially available ELISA kits. The main outcome measures are changes in renal function levels within the first 28 days following MSC infusion.

Ethics and dissemination The study was approved by the Ethics Committee of the Chinese PLA General Hospital. The findings of the study will be disseminated through public and scientific channels.

Trial registration number NCT04194671.

\section{INTRODUCTION}

Acute kidney injury (AKI) is a global public health issue characterised by a sudden loss of kidney function, which has an important impact on morbidity and mortality. ${ }^{1-3}$ Higher serum creatinine is associated with an increased risk for mortality in patients with AKI. Patients with severe AKI, that is, those with either a doubling of baseline creatinine within 48 hours or a urine output of less than $0.5 \mathrm{~mL} / \mathrm{kg} /$ hour for more than 12 hour, are more likely to die than patients with mild AKI. ${ }^{4-6}$ A variety of factors, such as surgery, hypoxia, drugs, sepsis, inflammation,
Strengths and limitations of this study

- The main strength of our study is the number of treatments (two infusions), which may improve the efficacy of mesenchymal stem cells.

- The second strength is the rigorous design of inclusion and exclusion criteria for severe acute kidney injury to minimise sample heterogeneity to the greatest extent.

- The third strength is that our primary outcome measure is an objective measure of creatinine during the intervention period and follow-up periods to assess the change in renal function.

- A major limitation of this study lies in a small sample size due to the nature of exploratory clinical trials.

mechanical trauma, catheter-based interventions, ${ }^{7}$ haemodynamic instability and post-transplantation procedures, can cause AKI. ${ }^{8}$ AKI is mainly caused by ischaemiareperfusion injury (IRI), infection, and drugs and AKI may progress to chronic kidney disease (CKD) or even end-stage renal disease. ${ }^{910}$ Approximately, 25\% of AKI survivors will develop CKD. ${ }^{11}$ The effects of treatments for AKI, including symptomatic and supportive treatments, remain insufficient. Therefore, it is important to develop an effective treatment strategy for treating severe AKI.

The pathogenesis of AKI includes acute tubular injury, reactive oxygen species (ROS) generation, inflammatory response activation, tubular epithelial cell apoptosis, inflammatory cell infiltration and extensive release of proinflammatory cytokines. ${ }^{12-16}$ Therefore, reducing the immune response and ameliorating kidney tissue damage is a potential therapeutic approach for AKI.

Mesenchymal stem cells (MSCs) have been used in cell-based therapies for many human diseases. ${ }^{17}$ The therapeutic effects of MSCs 
Table 1 The characteristics of all clinical trials registered at clinicalTrial involving MSC-treated AKI

\begin{tabular}{|c|c|c|c|c|}
\hline Registration number & Status & Study title & Interventions & Country \\
\hline NCT01275612 & Withdrawn & $\begin{array}{l}\text { Mesenchymal stem cells in cisplatin-induced acute renal } \\
\text { failure in patients with solid organ cancers }\end{array}$ & MSC infusion & Italy \\
\hline NCT01602328 & Terminated & $\begin{array}{l}\text { A study to evaluate the safety and efficacy of ac607 for the } \\
\text { treatment of kidney injury in cardiac surgery subjects }\end{array}$ & AC607 & USA \\
\hline NCT04194671 & $\begin{array}{l}\text { Not yet } \\
\text { recruiting }\end{array}$ & $\begin{array}{l}\text { Clinical trial of mesenchymal stem cells in the treatment of } \\
\text { severe acute kidney injury }\end{array}$ & $\begin{array}{l}\text { Mesenchymal } \\
\text { stem cells }\end{array}$ & China \\
\hline NCT04445220 & Recruiting & $\begin{array}{l}\text { A study of cell therapy in COVID-19 subjects with acute kidney } \\
\text { injury who are receiving renal replacement therapy }\end{array}$ & SBI-101 & USA \\
\hline
\end{tabular}

AC607, a kind of allogeneic MSCs products; AKI, acute kidney injury; MSC, mesenchymal stem cell; SBI-101, an extracorporeal stromal cell therapeutic.

are due to paracrine effects. MSCs have been proposed to have immunomodulatory, anti-inflammatory, antioxidative, antiapoptotic and reparative properties. ${ }^{18} 19$ MSCs significantly attenuate acute IRI in an animal model. ${ }^{20-25}$ Therefore, the clinical application of MSCs for AKI treatment is promising. ${ }^{17}$

Several clinical studies have been conducted (table 1). ${ }^{26-29}$ The results from clinical trials of MSC therapy for AKI following cardiac surgery suggested that MSC therapy prevented the deterioration of renal function after cardiac operation, reduced hospital stay and the need for readmission and improved long-term outcomes of renal function. ${ }^{26}{ }^{28}$ However, another clinical trial demonstrated that allogeneic MSC transfusion after cardiac surgery did not decrease the time to recovery of kidney function. ${ }^{27}$ Given that it is still not known whether MSCs can improve the renal function of patients with AKI and because severe AKI has a high mortality, these results prompted us to design a randomised clinical trial (RCT) to test the safety and efficacy of MSC therapy for AKI.

\section{METHODS AND ANALYSIS}

\section{Study design}

We designed a patient-blinded, randomised, parallel placebo-controlled clinical trial among patients with severe AKI; we will randomly assign patients to receive either MSCs at a dose of $1 \times 10^{6}$ cells $/ \mathrm{kg}$ or placebo at day 0 and day 7 . The total number of cells will be no more than $1 \times 10^{8}$ per person. This single-centre study will be conducted in the Department of Nephrology, the First Medical Centre, Chinese PLA General Hospital, Chinese PLA Institute of Nephrology, Beijing, China. An overview of the study is presented in figure 1. Any methodological changes in the study design or sample size, which may potentially affect the patients' safety or efficacy results, will be validated by the ethics committee before implementation.

\section{Study participants}

Participants who meet the following criteria will be included: (1) severe AKI, defined as a more than twofold increase in serum creatinine level compared with baseline creatinine level within 48 hours and/or urinary output consistently $<0.5 \mathrm{~mL} / \mathrm{kg} /$ hour over 12 hours; the lowest serum creatinine value in the 3 months preceding inclusion was taken as the baseline value. If data are not available, the serum creatinine baseline value was estimated using the Modification of Diet in Renal Disease study (MDRD) equation assuming that estimated glomerular filtration rate (eGFR) was $75 \mathrm{~mL} / \mathrm{min}^{*} 1.73 \mathrm{~m}^{2}$; (2) age between 18 and 65 years; (3) willingness to give written informed consent or having a legally acceptable representative willing to provide consent and (4) ability to comply with procedures and study visit schedule, including postdischarge follow-up.

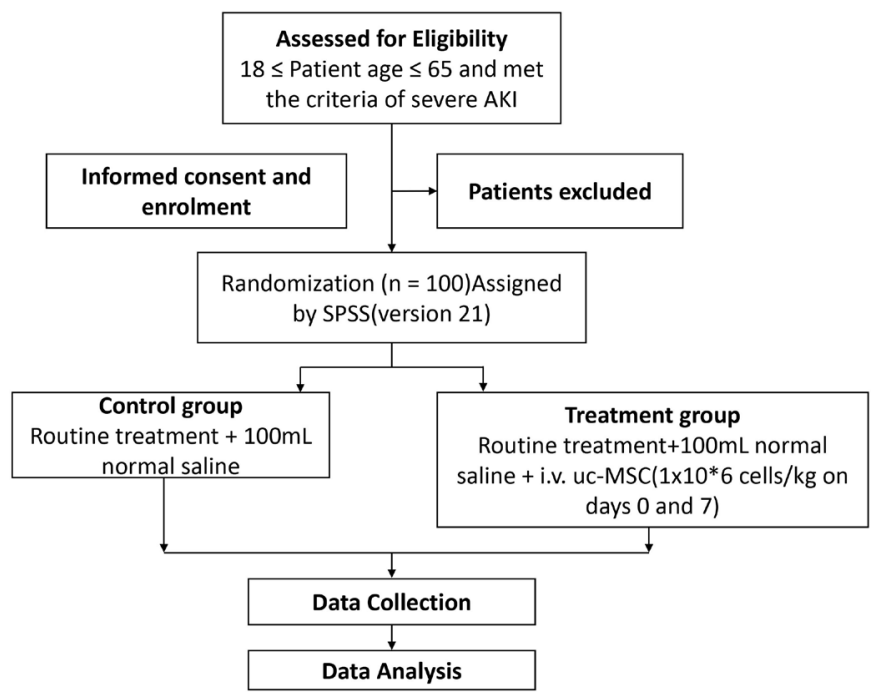

Figure 1 Flowchart of the efficacy of uc-MSC transfusion for patients with severe AKI. AKI, acute kidney injury; ucMSC, umbilical cord mesenchymal stem cell. 
Participants who meet any of the following criteria will be excluded: (1) AKI due to renal obstruction, glomerulonephritis, lupus nephritis, antineutrophil cytoplasmic antibody-related nephritis, antiglomerular-basement membrane antibody-mediated nephritis, cryoglobulinemia, thrombotic microangiopathy and purpura nephritis; (2) pregnancy or lactation; (3) allergic constitution; (4) previous haematopoietic stem cell transplantation or solid organ transplantation; (5) a history of benign or malignant tumours; (6) life expectancy less than 3 months; (7) known end-stage liver disease; (8) uncontrollable infection; (9) aged younger than 65 years old with an eGFR less than $60 \mathrm{~mL} / \mathrm{min} / 1.73 \mathrm{~m}^{2}$; (10) severe pulmonary dysfunction; (11) severe cardiac dysfunction, left ventricular ejection fraction less than $40 \%$, or severe arrhythmia; (12) haemodynamic instability; (13) organ failure affecting more than two nonrenal organs; (14) vasculitis of any cause; (15) a history of chronic systemic infection of any cause; (16) investigator belief that the subject may need to increase the vasopressor dose to achieve and/or maintain haemodynamic stability; (17) systemic immunosuppressive therapy that has not been stabilised for more than 4 months, or, in the case of chronic corticosteroid therapy, a dose of $>15 \mathrm{mg}$ /day of prednisone or the equivalent within the past 30 days; (18) platelet count $<25000 / \mu \mathrm{L}$ or other severe haematological abnormalities causing the subject to be at risk of death; (19) mechanical ventilation requirements and (20) participation in other clinical trials.

\section{Ethics and informed consent}

The current trial was approved by the Ethics Committee of the Chinese PLA General Hospital in April 2019 (file number: S2019-067-01). This study is registered at ClinicalTrials.gov. Informed consent (see online supplemental file 1) received prior to study inclusion by the investigating physician is required for this type of study according to Chinese law. Precautions will be taken to ensure the confidentiality of participants' information during and after the clinical study.

\section{Randomisation and allocation concealment}

The present study will be a patient-blinded parallel RCT. Subjects will be numbered in chronological order. A random number sequence generated by SPSS V.21.0 before study commencement will be used for patient randomisation. These generated numbers will be stored in opaque sealed envelopes which are sequentially numbered, and once generated, the list will remain unchanged. Independent drug administrators will store the envelopes, which will be concealed from participants and care providers until data analyses. Patients will be randomised 1:1 to either the MSC treatment group or the placebo control group according to their assigned random number.

\section{Intervention}

The recruitment of participants will take place in the Department of Nephrology of the Chinese PLA General Hospital. Investigators will explain the study protocol to potential participants in detail. If the patient decides to participate in this study, they will be required to sign a consent form. The efficacy and safety of MSCs will be compared between the treatment group and the control group. The control group will receive only $100 \mathrm{~mL}$ normal saline, while the treatment group will receive MSCs $+100 \mathrm{~mL}$ normal saline. Patients in both groups will receive conventional therapy as recommended by the attending physician according to the KDIGO Clinical Practice Guideline for AKI, including the removal of reversible causes, the maintenance of a stable internal environment, nutritional support, anti-infective treatment, the prevention and treatment of complications, rehydration and renal replacement therapy (RRT). Passage 4 umbilical cord MSCs (uc-MSCs) will be used. These MSCs will be manufactured by a Good Manufacturing Practicecertified company in China (Regend Therapeutics). Patients in the treatment group will receive two intravenous infusions at a dose of $1 \times 10^{6}$ cells $/ \mathrm{kg}$ on days 0 and 7 . The maximum dose of MSCs per person will not exceed $1 \times 10^{8}$ cells. The length of the infusion will be more than $30 \mathrm{~min}$. During the infusion, vital signs will be monitored continuously. The timing of treatment will be as soon as possible, and ideally within 72 hours of a clinical diagnosis of severe AKI. We will allocate other medications continuously used to treat concurrent diseases, and their volume and dosing will remain unchanged throughout the therapeutic intervention. Medication administration will be recorded in a case report form (CRF). The criteria for discontinuing the allocated interventions for a given trial participant were: (1) serious adverse events (SAEs); (2) serious deviations occurred in implementation and (3) China Food and Drug Administration's suspension of trials for some reason.

\section{Blinding}

Similar opaque infusion bags with different marks and black infusion lines will be used for both groups to ensure identical appearances. Independent drug administrators who receive group information based on the random number will inform dosing nurse and investigators responsible for recruiting participants of the group allocation. Then, the mixture of drugs will be prepared, and the bags will be labelled as A or B by the dosing nurse in a separate room. The participants will be blinded to the intervention allocation and the statistical analysts. Investigators and the clinical team looking after the patient (doctors, nurses and so on) are unblinded. Unblinding conditions are only permissible in case of medical emergency.

\section{Study outcomes}

The primary outcome is the difference in the renal function (creatinine) between the two groups (MSC treatment group vs placebo control group) within 28 days after 
receiving MSC/placebo treatment. These lab measurements were analysed at the Central Clinical Chemistry Laboratory, Chinese PLA General Hospital.

The secondary endpoints are as follows:

1. Overall survival within 28 days after receiving MSC/ placebo treatment.

2. Overall survival within 3 months after receiving MSC/ placebo treatment.

3. Dependence on RRT within 3 months after receiving $\mathrm{MSC} /$ placebo treatment.

4. Complete renal recovery within 3 months after receiving MSC/placebo treatment (complete recovery refers to survival, free of RRT and the serum creatinine (SCR) decreased to no more than 1.5 times of the baseline level. Baseline creatinine was defined as the lowest serum creatinine value in the 3 months preceding inclusion; if data are not available, the serum creatinine baseline value was estimated using the MDRD equation assuming that eGFR was $75 \mathrm{~mL} / \mathrm{min}^{*} 1.73 \mathrm{~m}^{2}$.).
5. Partial renal recovery within 3 months after receiving MSC treatment (partial recovery refers to survival, free of RRT and the SCR 1.5 times higher than the baseline level of creatinine).

6. ICU and hospitalisation duration of stay among survivors and those who died within 3 months after receiving MSC treatment.

7. Adverse events within 3 months after receiving MSC treatment.

\section{Follow-up}

The trial follow-up schedule is shown in table 2. At the screening stage, the study participants will undergo assessment for the inclusion and exclusion criteria alongside a full medical history and physical examination. Follow-up visits will be conducted during hospitalisation and after discharge. In the intervention period (days 0-28), data will be recorded at 1, 3, 7, 14 and 28 days. After discharge, follow-up will take place during outpatient clinic visits at

\begin{tabular}{|c|c|c|c|c|c|c|c|c|c|c|}
\hline & \multirow{2}{*}{$\begin{array}{l}\text { Enrolment } \\
\text { Admission }\end{array}$} & \multicolumn{6}{|c|}{ Intervention (28 days) } & \multicolumn{3}{|c|}{ Follow-up } \\
\hline & & D 0 & D 1 & D 3 & D 7 & D 14 & D 28 & M 2 & M 3 & Y 3 \\
\hline Medical history-taking & $x$ & & & & & & & & & \\
\hline Physical examination & $x$ & $x$ & $x$ & $x$ & $x$ & $x$ & $x$ & $x$ & $x$ & \\
\hline Screening, entry standard audit & $x$ & & & & & & & & & \\
\hline Informed consent & $x$ & & & & & & & & & \\
\hline Randomisation & $x$ & & & & & & & & & \\
\hline Blood routine & $x$ & & $x$ & $x$ & $x$ & $x$ & $x$ & $x$ & $x$ & \\
\hline Blood biochemistry & $x$ & & $x$ & $x$ & $x$ & $x$ & $x$ & $x$ & $x$ & \\
\hline Urine test & $x$ & & $x$ & $x$ & $x$ & $x$ & $x$ & $x$ & $x$ & \\
\hline Coagulation function & $x$ & & & & & & & & & \\
\hline Serology tests & $x$ & & & & & & & & & \\
\hline Cardiac markers & $x$ & & & & & & & & & \\
\hline ECG & $x$ & & & & & & & & & \\
\hline MSC infusion & $x$ & & & & $x$ & & & & & \\
\hline PRA & $x$ & & & & & & $x$ & & & \\
\hline Tumour marker & $x$ & & & & & & & & & \\
\hline Sample collection & $x$ & & & $x$ & $x$ & & $x$ & & & \\
\hline SOFA & $x$ & & & & & & & & & \\
\hline APACHEII & $x$ & & & & & & & & & \\
\hline Risk factor assessment & $x$ & & & & & & & & & \\
\hline Aetiology assessment & $x$ & & & & & & & & & \\
\hline Concomitant diseases & $x$ & & & & & & & & & \\
\hline Renal replacement therapy & $x$ & $x$ & $x$ & $x$ & $x$ & $x$ & $x$ & & & \\
\hline Blood transfusion & $x$ & $x$ & $x$ & $x$ & $x$ & $x$ & $x$ & & & \\
\hline AEs and handling & $x$ & $x$ & $x$ & $x$ & $x$ & $x$ & $x$ & $x$ & $x$ & \\
\hline Tumorigenesis & & & & & & & & & & $x$ \\
\hline
\end{tabular}

AEs, adverse events; APACHE II, Acute Physiology and Chronic Health Evaluation II; MSC, mesenchymal stem cell; PRA, panel-reactive antibody; SOFA, Sequential Organ Failure Assessment. 
2 months and 3 months for both study groups. We will establish contact via telephone and ask whether tumours occurred after hospital discharge for 3 years.

\section{Blood and urine sample collection}

Urine and blood samples for the biomarker assay will be collected at enrolment and on days 3, 7 and 28 . The samples will be frozen at $-80^{\circ} \mathrm{C}$ immediately after processing until use. We plan to use these samples to detect kidney injury biomarkers, renal repair biomarkers, inflammatory markers, immunomodulatory factors and fibrosis markers.

\section{Sample size}

Due to the exploratory nature of the study, no sample size calculation was performed. Assuming a $20 \%$ dropout rate, the final sample size is set to 50 participants in each group.

\section{Clinical assessments and data collection}

Clinical information, including age, medical history, comorbidities, Sequential Organ Failure Assessment score, Acute Physiology and Chronic Health Evaluation II score and the severity of AKI, will be documented within the clinical information system of the hospital after enrolment. The type and duration of RRT, the use of vasoactive drugs and the number of blood products will be recorded at all interviews from the beginning of the study by the investigating physician. Vital signs, including breathing, body temperature, pulse, blood pressure and adverse events, used for safety assessment, will be measured during human MSC infusion. Any adverse events, such as fever, itching, headache, vomiting and weakness, will be reported to the Medical Ethics Committee of the Chinese PLA General Hospital within 15 days.

Routine blood, urine test, biochemical, renal function (serum creatinine, blood urea nitrogen and cystatin $\mathrm{C}$ (CysC) levels), panel-reactive antibody, cardiac markers (creatine kinase, lactate dehydrogenase, phosphocreatine kinase isoenzyme), blood coagulation, ECG, tumour marker and serological tests will be performed. These routine lab measurements were analysed at the Central Clinical Chemistry Laboratory, Chinese PLA General Hospital.

For cases withdrawn from the study or dropped out for follow-up, the investigator should actively take measures to complete the last test as much as possible in order to analyse its efficacy and safety and take corresponding treatment measures. For all dropped cases, we will document the reason for the case drop on the CRF.

\section{Confidentiality}

Information will be entered into a standardised electronic CRF from the clinical information system of the hospital, stored in Department of Nephrology, the First Medical Centre, Chinese PLA General Hospital with a password protected hard disk. All data will be handled and processed anonymously to ensure the confidentiality of the individuals. Only the researchers will have access to the final trial dataset.

\section{Statistical analysis}

Statistical analyses will be performed using SPSS V.21. If the disease progressed rapidly after randomisation so that the patient receives only one infusion or no infusion, we will still include them for the primary analysis, as allocation to MSC treatment or placebo control arm could influence the likelihood that patients receive a transfusion. Continuous variables will be summarised as means with SD if normally distributed and medians with first and third quartiles otherwise. We will use the onesample Kolmogorov-Smirnov test to check whether the data are normally distributed. For the effectiveness analysis, missing data will be imputed using last observation carried forward, and for patients with missing data due to death, missing data will be imputed using the worst possible value (in the dataset). For the safety analysis, it will be conducted with the raw data. Because of the small sample sizes, we considered subgroup analyses were less suitable in the present study. We planned no subgroup analyses prespecified based on baseline characteristics. However, a posthoc subgroup analysis will only analysed if there was no difference between the two groups, in order to provide the basis for our next research. Due to the exploratory nature of the study, a forInterventtionsmal data monitoring committee or interim analyses were not considered. However, data monitoring will be conducted by the research team regularly.

For continuous variables, such as hospital length of intensive care unit (ICU) stay and total hospital length of stay, we will use non-parametric tests to analyse the differences between groups. Continuous variables such as creatinine, GFR, CysC, urine volume, urea nitrogen and all kinds of biomarkers are repeat-measurement data. We will develop a statistical analysis plan in consultation with a statistician, taking into account the repeated nature of the measurements. Data for categorical variables, including the survival of patients with severe AKI at 28 days and 3 months after receiving MSC, RRT dependence at 3 months, complete recovery rate, partial recovery rate and the incidence of adverse events, will be presented as proportions and compared using a $\chi^{2}$ test.

\section{Patient and public involvement}

The development of the research questions and outcome measures were not informed by patients' priorities, experience or preferences. Patients and public will not be involved in the design nor recruitment and conduct of study. Results will be disseminated to all study participants after study completion. The burden of the intervention was not assessed by the patients themselves.

\section{Safety consideration}

All necessary procedures and precautions will be taken to maximise participant safety. First, patients with higher potential risks will be excluded based on the exclusion 
criteria. Second, an infusion time of more $30 \mathrm{~min}$ was chosen to minimise the risk of infusion-related adverse effects. Third, during the infusion, vital signs will be continuously monitored. Fourth, once an adverse event occurs, SAEs considered to be related to the study procedure will be submitted to the principal investigator within 24 hours and to the ethics committee within 15 days of the completion of treatment. Then, the ethics committee will report the event to the Food and Drug Administration and the Provincial Health and Family Planning Commission of China. The patient will be followed up until the event has stabilised. No independent auditing of trial conduct is planned.

\section{ETHICS AND DISSEMINATION}

The study protocol and trial documents including the consent form and participant information sheet have been approved by the Ethics Committee of the Chinese PLA General Hospital. The findings of the study will be disseminated through scientific peer-reviewed journals as well as research conferences.

\section{DISCUSSION}

Severe AKI is associated with high mortality in patients. MSC therapy has shown the most attractive results in both preclinical and clinical research. At present, there are only three clinical trials registered in ClinicalTrials. gov of MSCs in the treatment of severe acute renal injury. Two of them have been completed, and one is recruiting subjects. Due to the opposite conclusions of the two trials and scarce evidence, more clinical trials are required to verify these inconsistent conclusions. Our study was designed to define the effects of uc-MSCs on severe AKI.

A strength of this study is that we will include only patients with AKI caused directly by renal tubular epithelial cell damage, and not those with AKI caused by glomerular or vascular disease, to ensure the homogeneity of the study sample. We will also exclude patients who are severely ill due to damage to other organs that may aggravate the patient's condition and possibly result in a higher mortality rate, which may mask the genuine action of MSCs.

A novel aspect of this study is the two intravenous infusions of MSCs on days 0 and 7 . When cells are injected into an injured environment, they face a harsh internal environment, including ROS and anoikis, which contribute to MSC apoptosis. ${ }^{30-32}$ Additionally, cell accumulation in the lung, liver and spleen leads to a small number of cells surviving to engraft injured kidneys. Due to the high risk of pulmonary embolism in bed-ridden patients, who are too ill to receive a large volume of cells that could accumulate in the lungs, a dose of $10^{6}$ MSCs per $1 \mathrm{~kg}$ will be used in this trial, which is half the number administered in other clinical trials. At the same time, considering the need for a sufficient dose of cells, an additional infusion will be conducted to increase the possibility of a good curative effect. MSCs will be administered by intravenous infusion, which is different from previous research. Compared with arterial infusion, intravenous infusion is more convenient and more easily accepted by patients.

Finally, our primary outcome measure is an objective measure of creatinine during the intervention period and follow-up periods to assess the change in renal function. The selection of these objective measures will make it possible to monitor continuous and dynamic changes in renal function and differences between the two groups.

However, the main limitation of our trial should be noted. A small sample size due to the nature of exploratory clinical trials needs to be further explored in the future.

\section{TRIAL STATUS}

Patient recruitment started on 31 December 2021 and will be completed on 31 December 2022.

Acknowledgements We thank Xiaowei Sun, Shuwei Duan and Quan Hong for their help and advice.

Contributors GC obtained the research funding and is the principal investigator. GC, YY and JG conceived and designed this study. YY drafted the manuscript. SW, WW, F-LZ, XW, SLia, ZF, SLin, LZ and XC all participated in the final design of the study. YY corrected this manuscript.

Funding This work is supported by the National Key R\&D Program of China (2018YFA0108803).

\section{Competing interests None declared.}

Patient consent for publication Not applicable.

Provenance and peer review Not commissioned; externally peer reviewed.

Supplemental material This content has been supplied by the author(s). It has not been vetted by BMJ Publishing Group Limited (BMJ) and may not have been peer-reviewed. Any opinions or recommendations discussed are solely those of the author(s) and are not endorsed by BMJ. BMJ disclaims all liability and responsibility arising from any reliance placed on the content. Where the content includes any translated material, BMJ does not warrant the accuracy and reliability of the translations (including but not limited to local regulations, clinical guidelines, terminology, drug names and drug dosages), and is not responsible for any error and/or omissions arising from translation and adaptation or otherwise.

Open access This is an open access article distributed in accordance with the Creative Commons Attribution Non Commercial (CC BY-NC 4.0) license, which permits others to distribute, remix, adapt, build upon this work non-commercially, and license their derivative works on different terms, provided the original work is properly cited, appropriate credit is given, any changes made indicated, and the use is non-commercial. See: http://creativecommons.org/licenses/by-nc/4.0/.

\section{ORCID iDs}

Yuanjun Yang http://orcid.org/0000-0003-2132-383X

Jianjun Gao http://orcid.org/0000-0001-9675-3071

Guangyan Cai http://orcid.org/0000-0001-8586-6353

\section{REFERENCES}

1 Yang X, de Caestecker M, Otterbein LE, et al. Carbon monoxide: an emerging therapy for acute kidney injury. Med Res Rev 2020;40:1147-1177.

2 Rewa O, Bagshaw SM. Acute kidney injury-epidemiology, outcomes and economics. Nat Rev Nephrol 2014;10:193-207.

3 Hoste EAJ, Kellum JA, Selby NM, et al. Global epidemiology and outcomes of acute kidney injury. Nat Rev Nephrol 2018;14:607-25.

4 Ishani A, Xue JL, Himmelfarb J, et al. Acute kidney injury increases risk of ESRD among elderly. J Am Soc Nephrol 2009;20:223-8. 
5 Hoste EAJ, Bagshaw SM, Bellomo R, et al. Epidemiology of acute kidney injury in critically ill patients: the multinational AKI-EPI study. Intensive Care Med 2015;41:1411-23.

6 Kaddourah A, Basu RK, Bagshaw SM, et al. Epidemiology of acute kidney injury in critically ill children and young adults. N Engl J Med 2017;376:11-20.

7 Thiele RH, Isbell JM, Rosner MH. AKI associated with cardiac surgery. Clin J Am Soc Nephrol 2015;10:500-14.

8 Rahman M, Shad F, Smith MC. Acute kidney injury: a guide to diagnosis and management. Am Fam Physician 2012;86:631-9.

9 Chawla LS, Eggers PW, Star RA, et al. Acute kidney injury and chronic kidney disease as interconnected syndromes. N Engl J Med 2014;371:58-66.

10 Molitoris BA, Okusa MD, Palevsky PM, et al. Design of clinical trials in AKI: a report from an NIDDK workshop. Trials of patients with sepsis and in selected Hospital settings. Clin J Am Soc Nephrol 2012;7:856-60.

11 Coca SG, Singanamala S, Parikh CR. Chronic kidney disease after acute kidney injury: a systematic review and meta-analysis. Kidney Int 2012;81:442-8.

12 Bonventre JV, Yang L. Cellular pathophysiology of ischemic acute kidney injury. J Clin Invest 2011;121:4210-21.

13 Lee K-H, Tseng W-C, Yang C-Y, et al. The anti-inflammatory, antioxidative, and anti-apoptotic benefits of stem cells in acute ischemic kidney injury. Int J Mol Sci 2019;20. doi:10.3390/ijms20143529. [Epub ahead of print: 19 Jul 2019].

14 Tammaro A, Kers J, Scantlebery AML, et al. Metabolic flexibility and innate immunity in renal ischemia reperfusion injury: the fine balance between adaptive repair and tissue degeneration. Front Immunol 2020;11:1346.

15 Gharaie Fathabad S, Kurzhagen JT, Sadasivam M, et al. T lymphocytes in acute kidney injury and repair. Semin Nephrol 2020;40:114-25.

16 Chen W, Li D. Reactive Oxygen Species (ROS)-Responsive Nanomedicine for Solving Ischemia-Reperfusion Injury. Front Chem 2020;8:732.

17 Shi Y, Wang Y, Li Q, et al. Immunoregulatory mechanisms of mesenchymal stem and stromal cells in inflammatory diseases. Nat Rev Nephrol 2018;14:493-507.

18 Özmert E, Arslan U. Management of retinitis pigmentosa by Wharton's jelly derived mesenchymal stem cells: preliminary clinical results. Stem Cell Res Ther 2020;11:25.

19 Redondo J, Sarkar P, Kemp K, et al. Dysregulation of mesenchymal stromal cell antioxidant responses in progressive multiple sclerosis. Stem Cells Trans/ Med 2018;7:748-58.
20 Perico L, Morigi M, Rota C, et al. Human mesenchymal stromal cells transplanted into mice stimulate renal tubular cells and enhance mitochondrial function. Nat Commun 2017;8:983.

21 Chen $\mathrm{H}-\mathrm{H}$, Lin K-C, Wallace CG, et al. Additional benefit of combined therapy with melatonin and apoptotic adipose-derived mesenchymal stem cell against sepsis-induced kidney injury. J Pineal Res 2014;57:16-32

22 Jiang MH, Li G, Liu J, et al. Nestin(+) kidney resident mesenchymal stem cells for the treatment of acute kidney ischemia injury. Biomaterials 2015;50:56-66.

23 Zhu G, Pei L, Lin F, et al. Exosomes from human-bone-marrowderived mesenchymal stem cells protect against renal ischemia/ reperfusion injury via transferring miR-199a-3p. J Cell Physiol 2019;234:23736-49.

24 Kim D-J, Moon J-Y, Kim S-M, et al. Substance P improves renal ischemia reperfusion injury through modulating immune response. Front Immunol 2020;11:600.

$25 \mathrm{Cao} \mathrm{H}$, Cheng Y, Gao H, et al. In Vivo Tracking of Mesenchymal Stem Cell-Derived Extracellular Vesicles Improving Mitochondrial Function in Renal Ischemia-Reperfusion Injury. ACS Nano 2020;14:4014-26.

26 Westenfelder C, Togel FE. Protective actions of administered mesenchymal stem cells in acute kidney injury: relevance to clinica trials. Kidney Int Supp/ 2011;1:103-6.

27 Swaminathan M, Stafford-Smith M, Chertow GM, et al. Allogeneic mesenchymal stem cells for treatment of AKI after cardiac surgery. $J$ Am Soc Nephrol 2018;29:260-7.

28 Tögel FE, Westenfelder $\mathrm{C}$. Kidney protection and regeneration following acute injury: progress through stem cell therapy. Am J Kidney Dis 2012;60:1012-22.

29 Miller BLK, Garg P, Bronstein B, et al. Extracorporeal stromal cell therapy for subjects with Dialysis-Dependent acute kidney injury. Kidney Int Rep 2018;3:1119-27.

30 Robey TE, Saiget MK, Reinecke H, et al. Systems approaches to preventing transplanted cell death in cardiac repair. J Mol Cell Cardiol 2008;45:567-81.

31 Song H, Cha M-J, Song B-W, et al. Reactive oxygen species inhibit adhesion of mesenchymal stem cells implanted into ischemic myocardium via interference of focal adhesion complex. Stem Cells 2010;28:555-63.

32 Chang W, Song B-W, Moon J-Y, et al. Anti-death strategies against oxidative stress in grafted mesenchymal stem cells. Histol Histopathol 2013;28:1529-36. 